\title{
Feasibility Analysis of UAV Operations for Monitoring of Infrastructure Networks: a Risk-based Approach*
}

\author{
S. Bertrand, N. Raballand, S. Lala and F. Viguier
}

\begin{abstract}
This paper proposes a method to analyze the feasibility of monitoring missions of infrastructure networks (eg. railways, roads, power lines, etc.) by Unmanned Aerial Vehicles (UAVs). The method consists in evaluating the risk at ground for people due to an UAV flying over each part of the network. Based on a risk categorization defined by probability and exposition time, several metrics are developed to analyze the feasibility of flights in terms of risk. More specifically, these metrics are related to the cumulative length of feasible flight segments and their connectedness. A decision making process is then defined to automatically classify all the lines of the network in a comprehensive and synthetic way. The whole method takes into account flight regulation and flight operation constraints as well as considerations relative to business interests in asset management of the infrastructure network. An illustration is proposed through a benchmark example on the French railway network.
\end{abstract}

\section{INTRODUCTION}

Infrastructure networks for public transportation (railways, motorways), electricity or gas transportation, etc. require regular monitoring operations. Airborne platforms provide a good mean to collect data over long distances, in complement to ground means, in order to check the state of the network and help to plan maintenance operations. In this context, Unmanned Aerial Vehicles (UAVs) offer a promising alternative to traditional aircraft (planes, helicopters) in terms of service availability and cost reduction. Nevertheless their regular operational use is still conditioned by being able to guarantee a sufficient level of safety and obtain flight authorizations. Safety methods have been developed and applied to UAVs to identify possible failure modes, quantify the risk level associated to the platform, and propose risk mitigation technologies or procedures [1][2][3]. Risk assessment methods have also been proposed in the literature to quantify the risk related to the mission. More specifically, a particular attention has been payed to risks due to UAV operations for people at ground [4][5] or, more recently by the authors, for users of road networks [6]. Evaluations have been performed for case studies representative of the inspection of one rail line [7] or one power line [8] by small fixed-wing UAVs. Quantified risk results have been obtained with a quite

*This work has been supported by the research partnership between SNCF Réseau, ONERA and Altametris.

S. Bertrand, N. Raballand and S. Lala are with ONERA - The French Aerospacelab, Palaiseau, France (sylvain.bertrand@onera.fr, nicolas.raballand@onera.fr, stephanie.lala@onera.fr).

F. Viguier is with Altametris, Saint-Denis, France (flavien.viguier@altametris.com). precise resolution at ground. Nevertheless, analysis of the complete infrastructure network has not been considered. On the contrary, other evaluations have been performed in the literature [9][10] over very wide areas (at the scale of a country and for High Altitude and Long Endurance UAVs) but with a low resolution at ground and without considering flight trajectories tailored to a particular mission such as the ones of interest in this paper.

The specificity of the problem of monitoring missions of infrastructure networks, as considered in this paper, is that risk evaluation must be performed along a very huge number of possible flight trajectories, over very long distances (for a network at the scale of a country) and with a precise ground resolution of the risk results. Solving such a problem would enable the asset manager to identify parts of the network over which UAV monitoring missions are feasible from this risk point of view, assess the business model behind UAV operations and help in the design and improvement of maintenance policies of the network. It will also enable to envisage permanent flight authorizations for identified feasible corridors over the network. Therefore the risk analysis methodology that has to be developed should encompass concerns relative to flight regulations, flight operations (deployment, flight endurance, etc.) and asset management. It must also provide comprehensive and synthetic results that could be used for decision making.

The method proposed in this paper aims at fulfilling these objectives. It is decomposed into four main steps, as illustrated in Figure 1. The first step, risk evaluation, consists in computing the probability and exposition time associated to the risk at ground for people. Based on these data, the second step performs a risk classification which is then used in the third step to identify feasible flight segments along the network. Cumulative length and connectedness of these segments are introduced as performance indexes which are used in the last step of the method, decision making, to automatically classify all the lines of the network in a comprehensive and synthetic way.

An illustration is proposed in the paper on the French railway network, over which UAV operations are already performed locally by Altametris, the drone subsidiary of SNCF Réseau (French railway company).

The organization of the paper follows the different steps of the method. Section II is devoted to risk evaluation and classification. Section III presents the identification process of flight segments and corresponding performance indexes. Rules and classes for decision making are defined in 
Section IV. Finally, before concluding remarks, Section V illustrates the proposed approach on the French railway network.

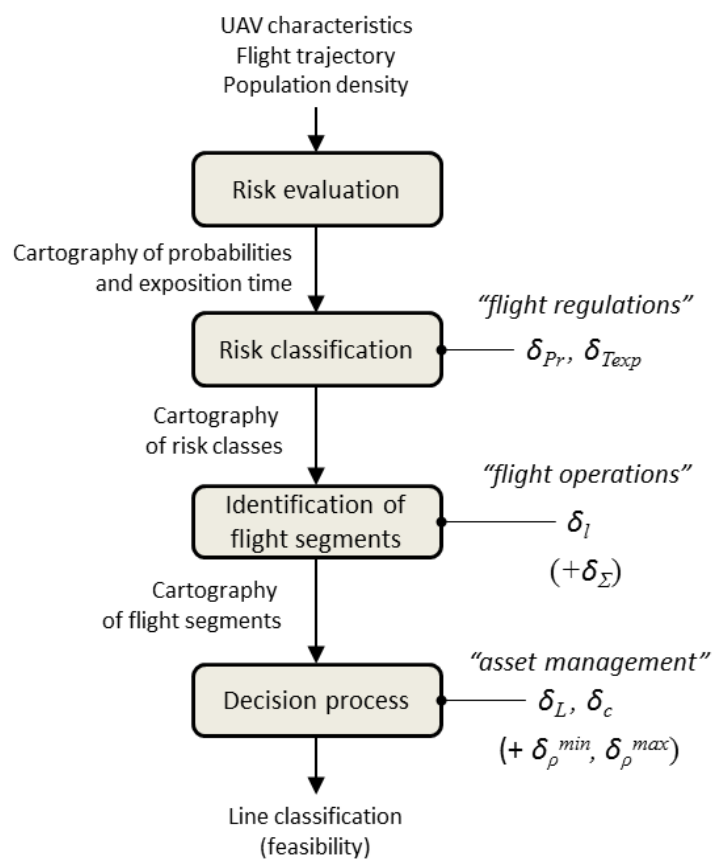

Figure 1. Flowchart of the method (to be applied to each line of the network)

\section{RISK EVALUATION AND CLASSIFICATION}

In this paper one considers the risk for people at ground induced by to the flight of a fixed-wing UAV over possibly inhabited areas. Models for quantitative risk evaluation are the ones used by the authors in [7] and are related to previous works in the literature such as [4]. They are briefly described in this section.

\section{A. Risk decomposition}

The risk of casualties (fatal injuries) for people at ground is evaluated by considering a succession of four events which are: a loss of control of the UAV leading to a descent to the ground, a non-controlled ground impact, a collision with someone, a fatal injury for impacted people. A probabilistic evaluation of this risk is made possible by taking into account the conditionality in the realization of these four events, leading to the following expression:

$$
\begin{aligned}
\operatorname{Pr}\{\text { casualty }\}= & \operatorname{Pr}\{\text { loss }\} \\
& . \operatorname{Pr}\{\text { impact } \mid \text { loss }\} \\
& . \operatorname{Pr}\{\text { collision } \mid \text { impact } \cap \text { loss }\} \\
& . \operatorname{Pr}\{\text { fatal injury } \mid \text { collision } \cap \text { impact } \cap \text { loss }\}
\end{aligned}
$$

A detailed description of the computation of all these terms can be found in [7]. Main engine failure is considered for $\operatorname{Pr}\{$ loss $\}$. The second term, $\operatorname{Pr}\{$ impact/loss $\}$, is computed over all the points of the impact area $A$ at ground. This area is defined by assuming a gliding descent to the ground and is therefore parametrized by the gliding range of the vehicle. Note that improved models can be used instead for this definition, such as the ones developed in [11]. The third term, $\operatorname{Pr}\{$ collision|impact $\cap$ loss $\}$, takes into account the surface of collision between the UAV and someone as well as the population density at ground. This density is based on [12][13] and is available with a geographical resolution of $\sim 130 \mathrm{~m} \times 90 \mathrm{~m}$ for France. Finally, the fourth and last term $\operatorname{Pr}\{$ fatal injury|collision $\cap$ impact $\cap$ loss $\}$ is evaluated by using the impact lethality model proposed in [9]. If we consider a commonly used way to express a risk, this final term represents the severity of the event as detailed in [7], whereas the first three terms are associated to its likelihood.

\section{B. Risk computation}

Equation (1) is used to compute the probability of casualties, at each instant of the UAV flight trajectory, and for all the points of the corresponding ground impact areas. Since a point at ground can belong to several impact areas corresponding to different instants $t$ of the trajectory, the maximum value of $\operatorname{Pr}\{$ casualty $\}(t)$, is computed over the whole trajectory duration. It is denoted by $\operatorname{Pr}_{s p a}^{\max }$ \{casualty\} (see definition in [7]) and its value at each possible point at ground can be visualized as a footprint map such as the one presented in Figure 2. In this figure, the blue line represents the flight trajectory of the UAV.

In addition, risk exposition time $T_{\text {exp }}$ is computed as the time duration each point at ground belongs to any possible crash area $A(t)$, for all time instant $t$ of the flight trajectory. This risk exposition time can also be visualized as a geographical map (see example in Figure 3).

The choice to merge the four terms of equation (1), covering likelihood and severity, into one single value $\operatorname{Pr}\{$ casualty $\}$ facilitates the comparison between the instantaneous risk (represented by this probability) and its accumulation over time (represented by $T_{\text {exp }}$ ). Moreover, it helps to define respective thresholds for risk classification, as detailed in the following section.

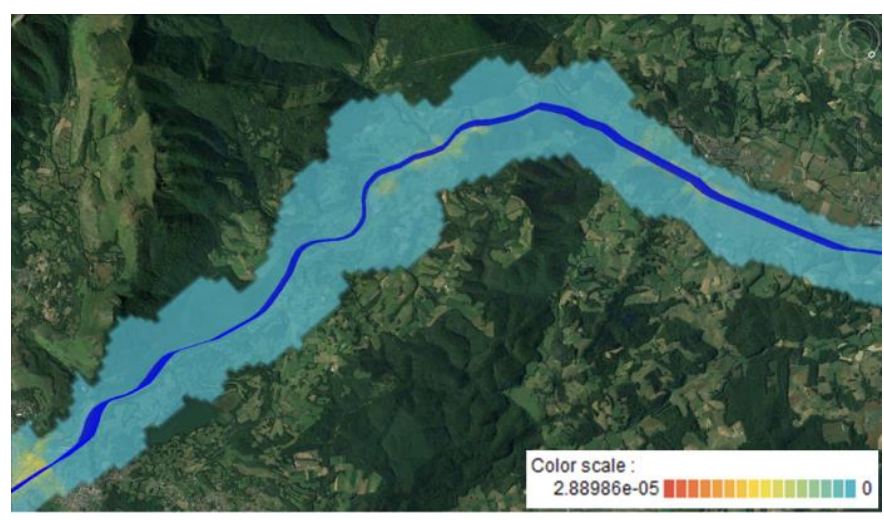

Figure 2. Example of geographical visualization of $\operatorname{Pr}_{s p a}^{\max }\{$ casualty $\}$ 


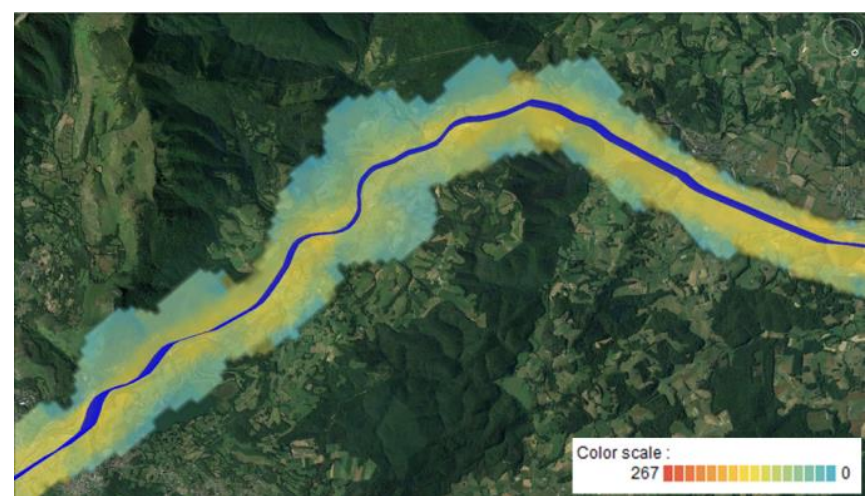

Figure 3. Example of geographical visualization of $T_{\exp }(\mathrm{s})$

\section{Risk classification}

Based on the values of $\left(\operatorname{Pr}_{s p a}^{\max }\{\right.$ casualty $\left.\}, T_{\text {exp }}\right)$ a classification of risk has been proposed by the authors in [7]. In this classification, analysis thresholds $\left(\delta_{P r}, \delta_{T e x p}\right)$ are defined to categorize points at ground into three classes: "high risk" ( $\mathrm{Pr}_{\text {spa }}^{\max }\{$ casualty $\} \geq \delta_{P r}$ and $\left.T_{\text {exp }} \geq \delta_{T e x p}\right)$, "low risk" $\left(\operatorname{Pr}_{\text {spa }}^{\max }\{\right.$ casualty $\}<\delta_{P r}$ and $\left.T_{\text {exp }}<\delta_{T e x p}\right)$, "medium risk" (other cases). For long range operations of UAVs, which are of interest in this paper, values $\delta_{P r}=10^{-7}$ and $\delta_{\text {Texp }}=60 \mathrm{~s}$ have been chosen, for conservativeness with respect to regulation considerations. Examples of Figure 2 and Figure 3 hence result in the risk classification map of Figure 4. This classification of risks enables a comprehensive interpretation of the quantitative evaluation done in terms of probability and exposition time. It will be used as a basis in the approach proposed in this paper for the feasibility analysis of UAV flights over an infrastructure network.

Remark 1: Note that any other approach that would lead to such a risk classification as a map along the trajectory of the UAV could be used instead. Therefore, in the proposed methodology, the flight segment identification and decision process steps are 'independent' from the nature of the risk evaluation and classification method.

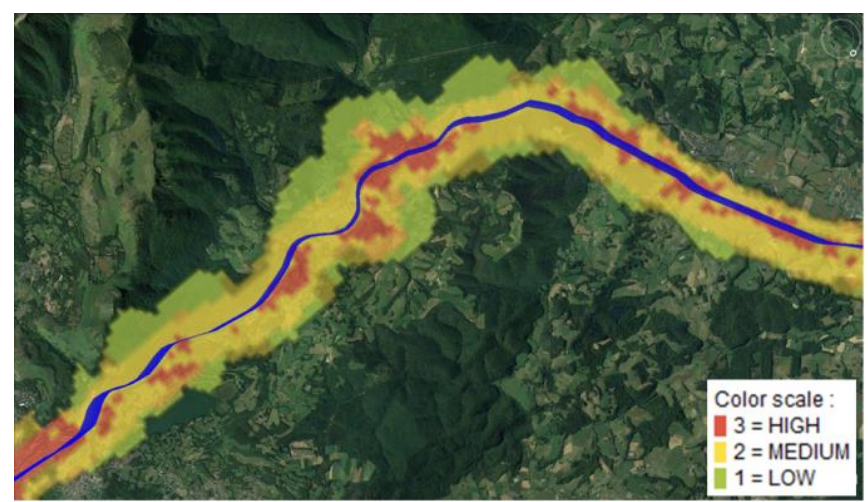

Figure 4. Example of geographical visualization of risk classes (red: high risk, yellow: medium risk, green: low risk) $\delta_{P r}=10^{-7}$ and $\delta_{T e x p}=60 \mathrm{~s}$

\section{IDENTIFICATION OF FLIGHT SEGMENTS}

In the case of large networks, eg. at the scale of a country, the amount of data to be processed for risk analysis can become very important and even prohibitive if one directly uses risk maps such as the ones described in the previous section. It can reasonably be assumed that the monitoring mission of a whole network can be decomposed into several sub-missions over 'sections' or 'lines' of the networks. To each line will correspond a long range trajectory (possibly several tens or hundreds of kilometers) that should be performed by one (or several) UAV(s). It is therefore of interest to dispose of high level indexes for risk analysis over long range trajectories. These indexes should be defined to provide quantitative answers to the questions arising in feasibility analysis of such monitoring missions. These questions of interest considered in this paper are:

- Is it possible to identify long flight segments along the lines of the network over which the risk level induced by UAV operations will be acceptable?

- Are such identified flight segments connected to each other, allowing long range UAV operations, or are they disconnected from each other, imposing several take-offs and landings to avoid flying over high risk areas?

Indexes are proposed in the next subsections to be able to answer these questions in a quantitative way.

\section{A. Linear repartition of risk classes}

Risk classes maps obtained from the previous risk classification step are processed in order to get information on the repartition of the risk classes along the flight trajectory. To do so, at each time step $t$ of the flight trajectory, the total surface corresponding to each risk class is computed inside the impact surface $A(t)$ (see illustration in Figure 5 at a given time step $t$ ). These data are then translated and represented as a function of the curvilinear abscissa along the flight trajectory leading to graphical representation such as the one of Figure 6 (top part of the figure).

Let us denote by $\Sigma(t)$ the total surface belonging to the high risk class inside $A(t)$ at time $t$ (see also Figure 5 for illustration). The representation of $\Sigma$ as a function of the distance $d$ along the flight trajectory is given by the red curve ('high risk' label) of Figure 6.

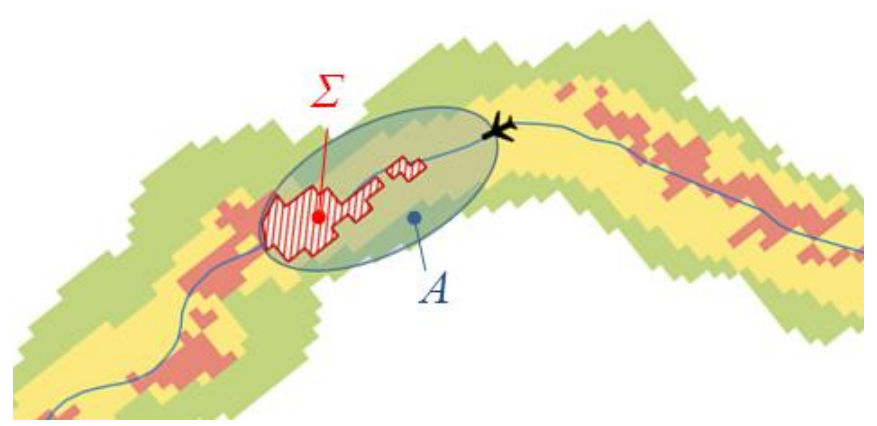

Figure 5. Surface $\Sigma$ of high level risk inside the impact footprint $A$ 


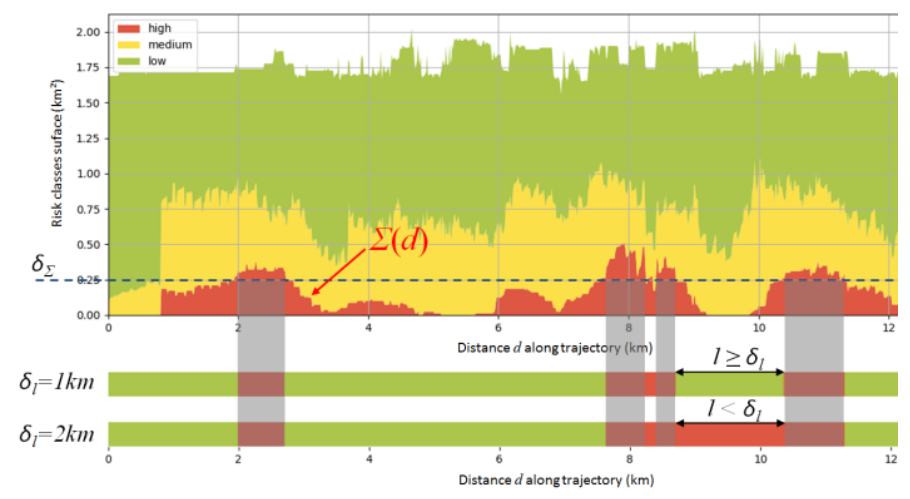

Figure 6. Linear repartition of risk classes along a given line of the network and identification of feasible flight segments

If $\Sigma$ is 'small', it will be considered that a slight local modification (eg. detour or flight altitude modification) of the flight trajectory will enable to avoid flying over highly risky areas. On the contrary if $\Sigma$ is 'too high', it will be considered that flying over the corresponding location is not feasible without requiring major changes in the flight trajectory, i.e. at least deviation outside the ground impact footprint, which is beyond the scope of this paper (see risk aware path planning methods for example [14][15]). Therefore it is of interest to define some threshold $\delta_{\Sigma}$ for the surface $\Sigma$. It is defined in terms of a percentage $N_{\%}$ of the maximum value $A^{\max }$ of $A(t)$ over the whole trajectory:

$$
\delta_{\Sigma}=N_{\%} \cdot A^{\max }=N_{\%} \cdot \max \{A(t) \mid t\}
$$

One major parameter influencing the size of the surface $A(t)$ is the altitude of the UAV (see model in [7]).

Therefore $A^{\max }$, and then $\delta_{\Sigma}$, can be defined by considering for example the maximum altitude authorized for the flight. In this paper, two values are chosen for $N_{\%}(20 \%$ and $30 \%)$ to check further the sensitivity of the proposed method with respect to this parameter. These values correspond to the possibility of performing a slight change in the altitude of the UAV, around its nominal cruise altitude, to modify the size of the impact area and possibly avoid high risky zones located in its periphery. For the flight trajectory considered as case-study (see Section V), the nominal altitude is $140 \mathrm{~m}$. A change down to $120 \mathrm{~m}$ would reduce the size of the ground impact area by a factor of $26.5 \%$ (according to the definition of the ground impact area used in [7] and its dependence to the altitude). Note that it only justifies the order of magnitude for values of $\delta_{\Sigma}$ tested in this paper $\left(N_{\%}=20 \%\right.$ and $30 \%$ ), since in many cases a change in the altitude of the UAV would not be sufficient to avoid impact risks in risky areas.

\section{B. Identification of feasible flight segments}

Feasible flight segments are identified as sections over which $\Sigma \leq \delta_{\Sigma}$ and the length of which is greater than a given minimum flyable distance $\delta_{l}$. This minimum length threshold is driven by flight operation concerns in the sense that flying over too short segments would require many take offs and landings and may be prohibitive from operational (deployment) and business point of views. The value of this threshold $\delta_{l}$ can be also chosen in compliance with the endurance of the UAV. Figure 6 provides an illustration of the identification process of flight segments. Two values of $\delta_{l}(1 \mathrm{~km}$ and $2 \mathrm{~km})$ are considered in the figure for illustration purpose leading to two bar charts where feasible flight segments are in green. As can be seen on this example, four segments do not satisfy the condition $\Sigma \leq \delta_{\Sigma}$ and are removed from the feasible ones (rectangles in grey). Other sections are then removed from the feasible flight segments if their lengths are lower than $\delta_{l}$.

The obtained bar charts indicating feasible flight segments are a valuable and comprehensive representation for the analysis of the feasibility of the monitoring mission over a line of the network. The analysis of the network can therefore be based on these flight segments. But due to the possibly high number of lines of the network, a visual analysis of all the corresponding bar charts can be too complex and prohibitive. It is hence important to derive other quantitative indexes on which the analysis could be based. Such indexes are proposed in the next subsection.

\section{Cumulative length and connectedness indexes}

A first index of interest that will be used in the decision making step is the sum $L$ of the lengths of all the flight segments identified along a given line of the network. This $L$-index can be expressed in kilometers or as a percentage of the length of the line.

A second index is the connectedness of the identified flight segments. It is denoted by $c$ and is defined as the ratio between values of $L$ obtained for 'extreme' $\delta_{l}$ values:

$$
c=\frac{L_{\left(\delta_{l}^{\max }\right)}}{L_{\left.\delta_{l}^{\min }\right)}}
$$

In this paper $\delta_{l}{ }^{\min }=2 \mathrm{~km}$ and $\delta_{l}{ }^{\max }=10 \mathrm{~km}$. With this definition, if all the flight segments are connected together the value of $L$ will be quite the same whatever the value of $\delta_{l}$ and $c$ will be close to 1 . On the contrary, if a lot of short and sparse segments are identified $L$ will decrease as $\delta_{l}$ increases and the $c$-index will be very small (close to zero). An example is given in Figure 7 in the case of two lines with respectively sparse flight segment $(c=0.12)$ and connected flight segments $(c=0.95)$.

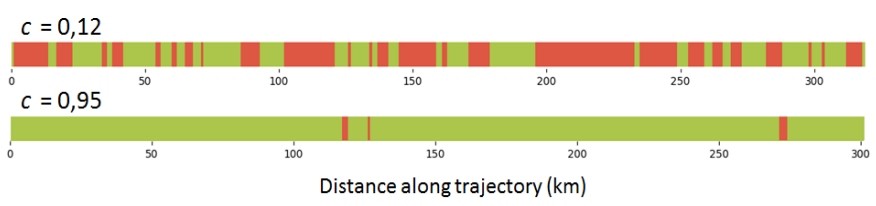

Figure 7. Connectedness of identified flight segments (in green): sparse flight segments (top) and connected flight segements (bottom) 


\section{DECISION PROCESS FOR NETWORK ANALYSIS}

For all the lines of the network, one disposes of two indexes which are the cumulative length $L$ of the identified flight segments and their connectedness index $c$. A line of interest would be a line for which $L$ is 'high enough' and $c$ is 'close to 1 as much as possible'. Two decision thresholds $\delta_{L}$ and $\delta_{c}$ are respectively introduced for this purpose. A line of interest will hence correspond to $L \geq \delta_{L}$ and $c \geq \delta_{c}$.

Note that the values of $\left(\delta_{P r}, \delta_{T e x p}\right)$ can be fixed by flight regulation considerations, $\delta_{l}$ by flight operation considerations and $\left(\delta_{L}, \delta_{c}\right)$ by asset management considerations. The main uncertainty hence remains in the choice of a value for $\delta_{\Sigma}$ which models the threshold on the acceptable part of the impact area belonging to the high risk class. Some guidelines for this choice have been discussed in Section III.A. Therefore two different values of $\delta_{\Sigma}$ will be considered, corresponding to $20 \%$ and $30 \%$ for $N_{\%}$, and the sensitivity of the results to this value will be directly considered in the decision process. For lines with small sensitivity to $\delta_{\Sigma}$, conclusions will be considered to be enough trustworthy. On the contrary, for lines exhibiting a great sensitivity to $\delta_{\Sigma}$, it will be considered that the proposed approach cannot be used to derive conclusions.

Therefore the proposed analysis will be based, for each line of the network, on the $L$ and $c$-indexes and their respective sensitivity ratios to the value of $\delta_{\Sigma}$ which are defined by

$$
\begin{gathered}
\rho_{L}=\frac{L_{\left(\delta_{\Sigma}=30 \%\right)}}{L_{\left(\delta_{\Sigma}=20 \%\right)}} \\
\rho_{c}=\frac{c_{\left(\delta_{\Sigma}=30 \%\right)}}{c_{\left(\delta_{\Sigma}=20 \%\right)}}
\end{gathered}
$$

Il will be assumed that small sensitivity to $\delta_{\Sigma}$ is obtained if these ratio are within a given interval $\left[\delta_{\rho}^{\min }, \delta_{\rho}{ }^{\max }\right]$ with $0<\delta_{\rho}^{\min } \leq 1 \leq \delta_{\rho}^{\text {max }}$.

For a given line of the network, the rules developed for decision making are presented in Table 1 regarding the $L$ index. The minimum and maximum values of $L$ obtained for the two hypothesis of $\delta_{\Sigma}$ are denoted by $L_{\min }=\min \left(L_{(\delta \Sigma=20 \%)}\right.$, $\left.L_{(\delta \Sigma=30 \%)}\right)$ and $L_{\max }=\max \left(L_{(\delta \Sigma=20 \%)}, L_{(\delta \Sigma=30 \%)}\right)$. A graphical example is presented on Figure 8 to illustrate these rules.

Regarding the $c$-index, the same rules as for the $L$-index are used. The corresponding table is not presented here for brevity reasons but is identical to Table 1 .

When the sensitivity to $\delta_{\Sigma}$ is small, the proposed approach enables to automatically identify with confidence lines of interest (Class 1) and to reject lines with no interest (Class 4). The classification process may suffer from thresholding effect for some lines, such as in case 2.1 (see Table 1 and Figure 8), and a decision cannot automatically be taken. Such lines are therefore classified into Class 2 meaning a potential interest that should be confirmed by performing a more precise analysis based on the bar charts of Section III.

When the sensitivity to $\delta_{\Sigma}$ is high, the proposed approach cannot be used to provide a conclusion enough trustworthy. In this case, lines are classified into Class 3. An exception is made when, despite the high sensitivity to $\delta_{\Sigma}$, the two values of $L$ (respectively $c$ ) are greater than the analysis threshold $\delta_{L}$ (respectively $\delta_{c}$ ), showing therefore a potential interest. Such lines are hence also classified into Class 2, for further investigations (case 2.2 in Table 1 and Figure 8).

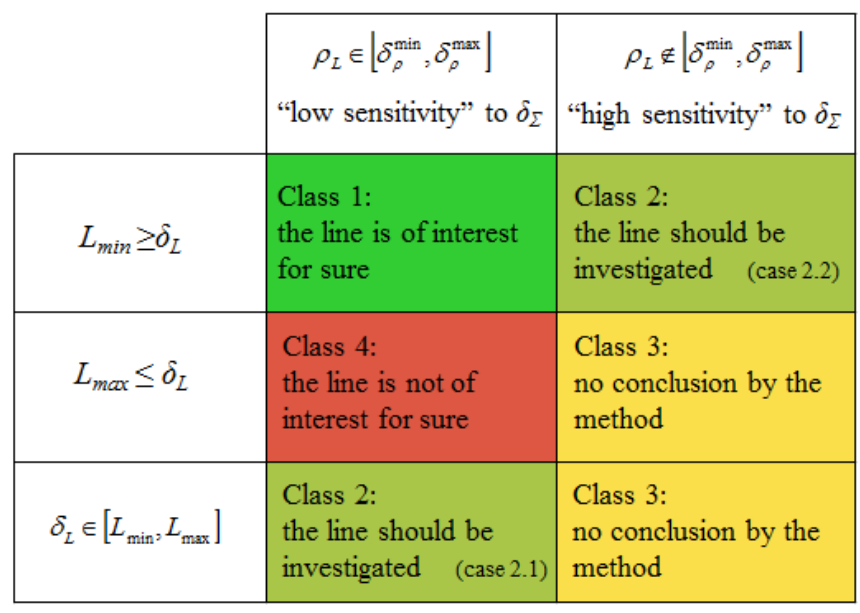

Table 1. Rules for decision making process regarding the $L$-index (The same table is used for rules regarding the $c$-index)

Remark 2: the case $L_{\min }=L_{\max }=\delta_{L}$ is classified into Class 1 .

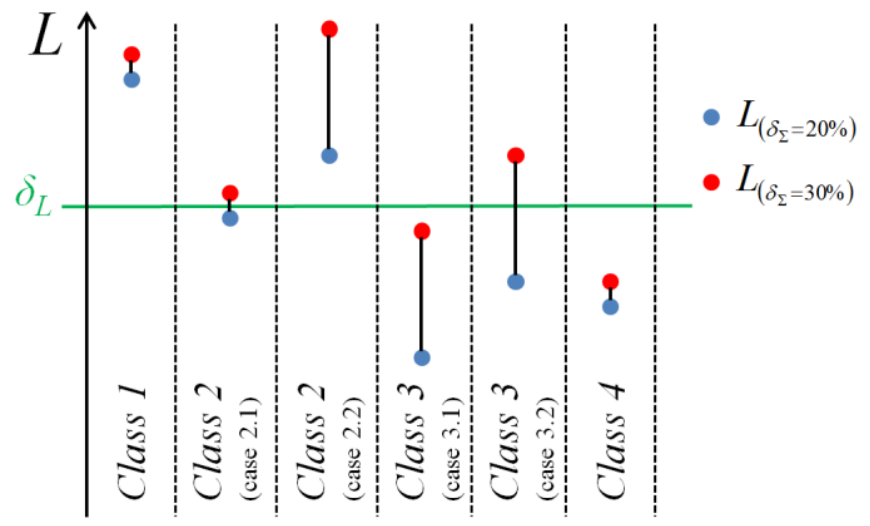

Figure 8. Classification rules: illustration example based on the $L$-index (condition on $\rho_{L}$ not taken into account quantitatively but illustratively by the length of the segments)

Remark 3: As previously stated, $L$ is computed for each line of the network for different values of the minimum length threshold for flight segments $\delta_{l} \in\{2,5,10\} \mathrm{km}$. The smaller $\delta_{l}$ is, the greater $L$ will be. This can easily be explained by the fact that, in addition to long flight segments which can be identified, other possible shorter flights segments can also be identified. In other words, only a subset of the short flight segments belongs to the set of the long flight segments. The example of Figure 6 illustrates this point. 
Therefore, in the absence of a choice given by operational considerations, the cumulative length $L$ that will be considered for feasibility analysis is chosen to be the one corresponding to the highest value of $\delta_{l}$, that is $\delta_{l}=10 \mathrm{~km}$. With this choice, a conservative feasibility analysis is performed in the sense that more flight segments can be identified on a line for other smaller values of $\delta_{l}$.

\section{CASE-STUDY: THE FRENCH RAILWAY NETWORK}

\section{A. Definition of case study}

The case study considered in this paper is the French railway network which is composed of more than 1000 rail lines for a total length of more than $36000 \mathrm{~km}$. Monitoring operations by UAVs will have to deal with many topography constraints as well as a wide variety of lines with surroundings diversely populated, either rural or urban.

A fixed-wing UAV is considered for this type of monitoring mission. The ground altitude and speed of the vehicle have been chosen to be respectively $140 \mathrm{~m}$ and $13.9 \mathrm{~m} / \mathrm{s}$. These values are representative of inspection missions of French railways currently performed by UAVs and are compliant with current French rules for BVLOS flights of UAVs.

\section{B. Risk evaluation and classification}

The risk evaluation step has been performed for all the lines of the network, considering a one-way flight ${ }^{1}$ above the lines. The risk evaluation tool is based on the BLADE simulation framework developed by ONERA. It provides $\mathrm{C}++$ fast-time simulation capabilities to compute the UAV flight trajectory and perform risk evaluation and classification at each time step. Identification of flight segments and decision making are then done in a post processing stage. The whole process is encapsulated into a multithread Python script which enables multi-runs to cover the whole network.

Computation time over the whole French railway network is one hour (standard PC). Figure 9 presents geographical maps obtained for the risk classes, such as the one of Figure 4 but over the whole network. As can be easily understood it represents a huge amount of data to be processed and analyzed. It hence justifies the development of the indexes and decision process proposed in this paper for a comprehensive feasibility analysis of the monitoring mission of each line of the network.

Although all the steps of the method have been applied to the whole French rail network, only a subset of rail lines is next considered in this paper for illustration. This benchmark is composed of the 80 longest lines of the network, each one

\footnotetext{
${ }^{1}$ Only one-way trajectories are considered in this paper, but it is worth noticing that outward and return trajectories may lead to different risks and should therefore be both considered.
}

being long of $100 \mathrm{~km}$ or more, representing a cumulative distance of more than $17000 \mathrm{~km}$. Figure 10 shows the length of each line considered in this benchmark, indexes being arbitrarily attributed after sorting the lines by their lengths. This choice of considering this "scaled" benchmark is motivated by the clarity of the presentation in this paper and by the fact the longest lines are the ones for which the business model of monitoring operations by UAVs is expected to be the most valuable.

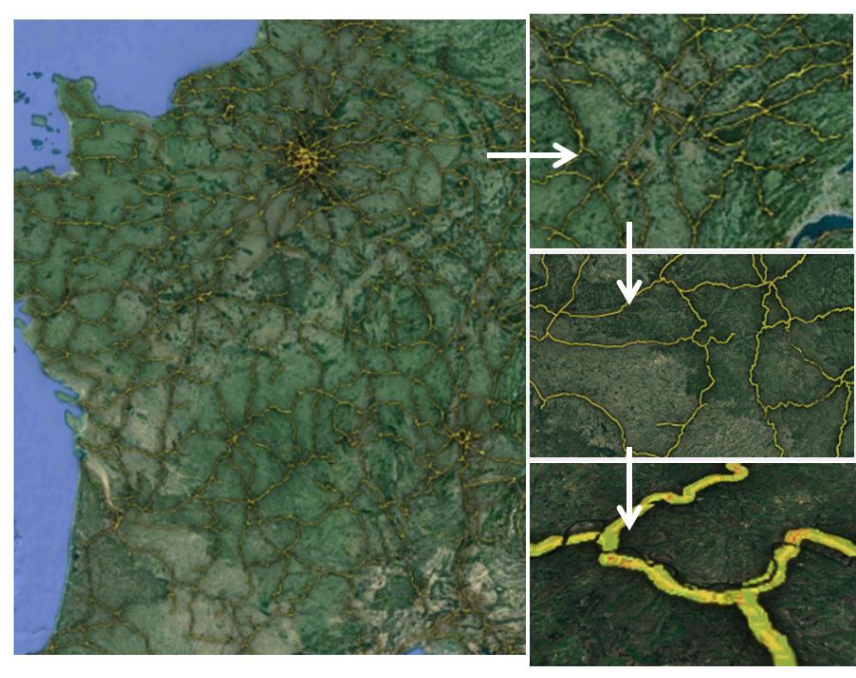

Figure 9. Risk evaluation performed over the whole French railway network: vizualization of risk classes (zooms)

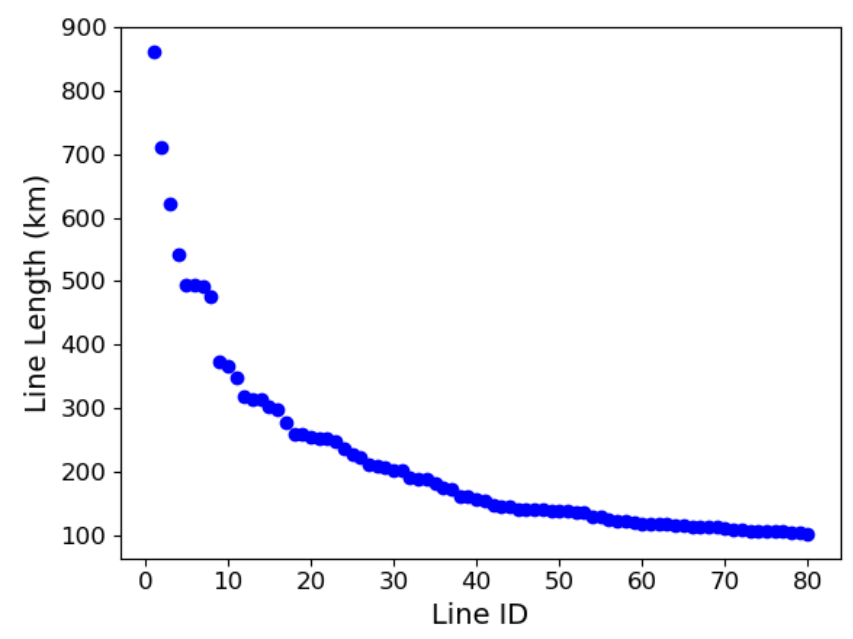

Figure 10. Length of each of the 80 longest rail lines of the French rail network considered as benchmark

\section{Feasibility analysis of monitoring missions}

The decision making step for feasibility analysis has been applied to obtain an automatic classification of the 80 lines of the aforementioned benchmark.

A representation of the $L$-index of each line is given in Figure 11. For a given line, the values of $L$ corresponding to the two $\delta_{\Sigma}$ assumptions are plotted as segments the length of which is representative of the value of the sensitivity ratio $\rho_{L}$. The 
green line corresponds to the value of the threshold $\delta_{L}=50 \mathrm{~km}$ used for decision making. The values of the sensitivity ratio $\rho_{L}$ is plotted in Figure 12, along with the decision thresholds $\delta_{\rho}{ }^{\min }=0.6$ and $\delta_{\rho}{ }^{\max }=1.4$ (green dotted lines). Rules defined in Table 1 have been applied on these $\left(L, \rho_{L}\right)$ data to produce an automatic classification of each line of the benchmark. This classification is graphically represented by the table of Figure 13. Each cell corresponds to a line, the number denoting its index and the color its class, as decided by the decision rules based on $\left(L, \rho_{L}\right)$.

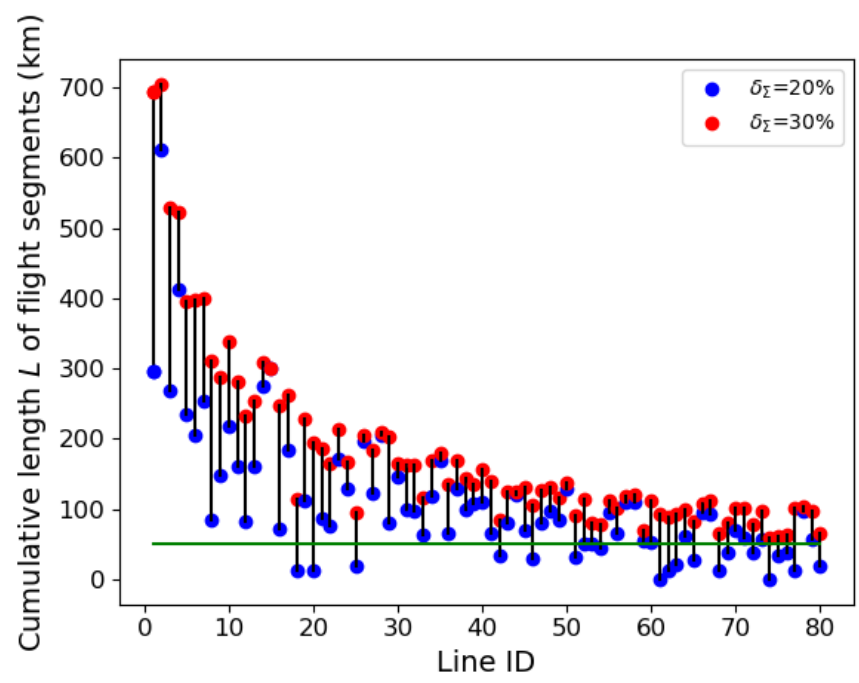

Figure 11. Values of the $L$-index for the 80 lines. Decision threshold $\delta_{L}=50 \mathrm{~km}$ (green line)

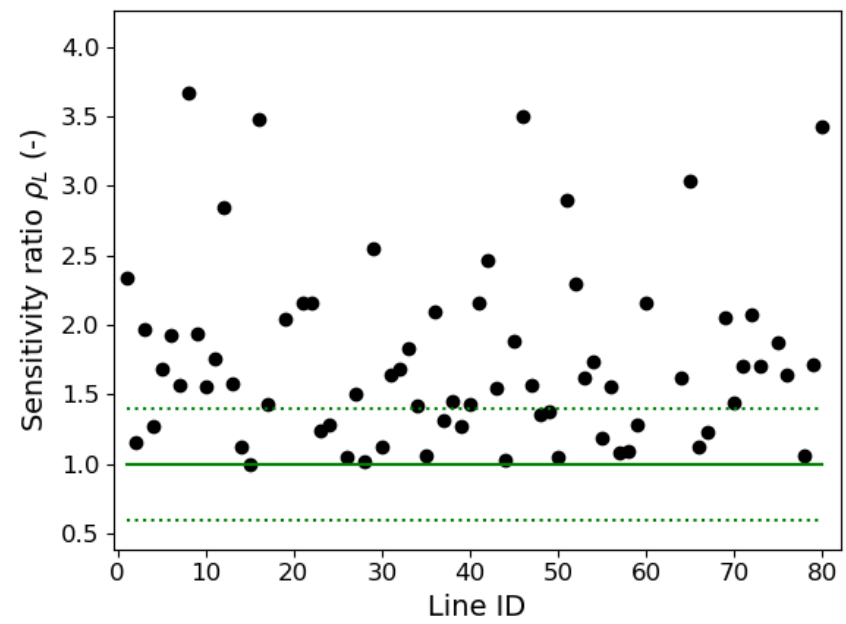

Figure 12. Values of the sensitivity ratio $\rho_{L}$ for the 80 lines. Decision thresholds $\delta_{\rho}{ }^{\text {min }}=0.6$ and $\delta_{\rho}{ }^{\max }=1.4$ (green dotted line)

The color code corresponds to the one used in Table 1 and is the following: green $=$ Class 1, pale green $=$ Class 2, yellow = Class 3, red = Class 4. As can be seen, 23 lines have been identified as feasible (i.e. Class 1) and 38 other lines show a potential interest that should be confirmed by an analysis of the associated bar chart data (i.e. Class 2). The classification into Class 3 concerns 19 lines for which the proposed approach is not able to provide automatically a conclusion due to the too high sensitivity to $\delta_{\Sigma}$. Further investigation is hence required for them to derive a trustworthy conclusion.

For the first 30 lines of the benchmark which have a length greater than $200 \mathrm{~km}$, it can be of interest to identify among Classl-lines the ones for which $L$ is greater than $100 \mathrm{~km}$. To do so, the decision process is run by considering the same $\left(L, \rho_{L}\right)$ data but with a decision threshold of $\delta_{L}=100 \mathrm{~km}$. The classification obtained in this case is presented in Figure 14. As can be seen, the 16 lines which still belong to Class 1 can be identified with high interest. Line 59 is now classified into Class 4 , because of a $L$-index below the threshold $\delta_{L}=100 \mathrm{~km}$ and a low sensitivity to $\delta_{\Sigma}$.

Note that the whole analysis has been performed by considering a minimum length threshold of $\delta_{l}=10 \mathrm{~km}$ for the identification of the flight segments. As already mentioned before, this choice leads to conservative results in terms of obtained cumulative distance of the flight segments since greater values of $L$ can be obtained for lower values of $\delta_{l}$.

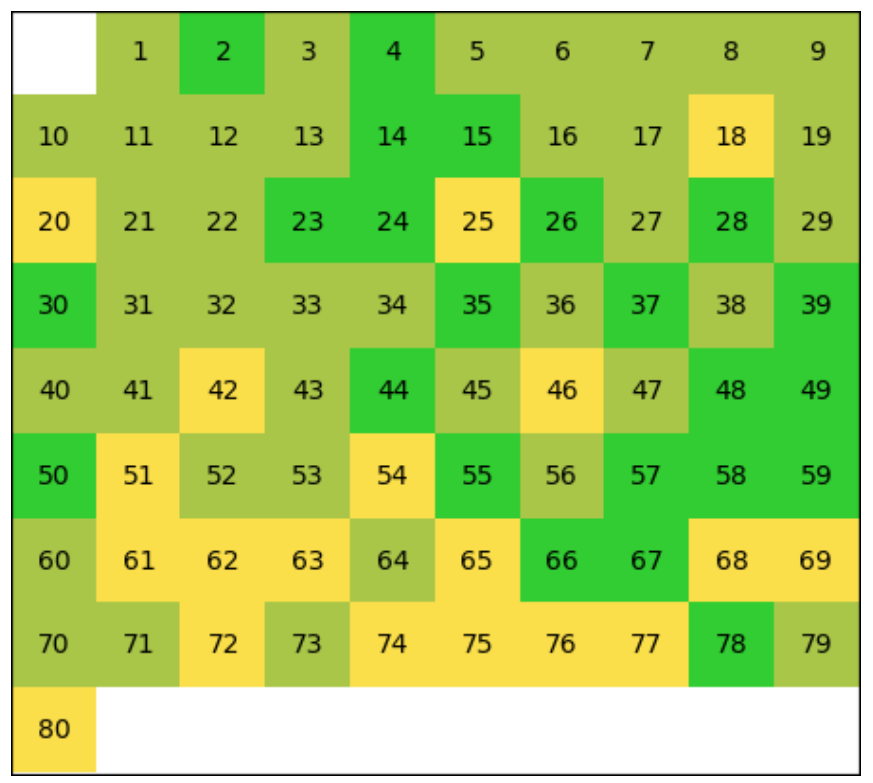

Figure 13. Classification of the lines based on $\left(L, \rho_{L}\right)$. Decision threshold $\delta_{L}=50 \mathrm{~km}$.

(see Table 1 for color code and definition of the classes)

The same analysis is performed regarding the connectedness index. The value of $c$ is plotted in Figure 15 and its corresponding sensitivity ratio $\rho_{c}$ in Figure 16. The classification obtained for $\left(c, \rho_{c}\right)$ using the same rules of Table 1 as for $\left(L, \rho_{L}\right)$ is presented in Figure 17. These additional results enable to improve the feasibility analysis of monitoring missions for the network. Indeed it provides information on the existence of long flight segments that could be inspected in a single UAV flight, hence simplifying operational aspects. A monitoring mission is then considered to be feasible for a line of Class 1 regarding the $L$-index (long cumulated distance), but a line of Class 1 both for the $L$ and $c$ indexes is an even better candidate for long range operations (long cumulated distance over connected flight segments). 


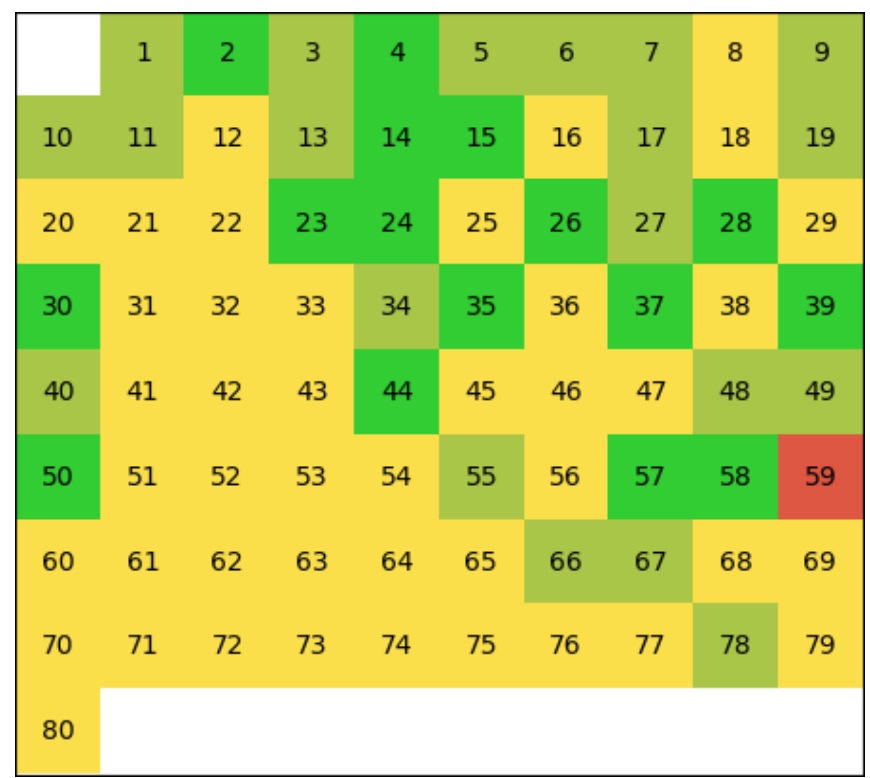

Figure 14. Classification of the lines based on $\left(L, \rho_{L}\right)$. Decision threshold $\delta_{L}=100 \mathrm{~km}$.

(see Table 1 for color code and definition of the classes)

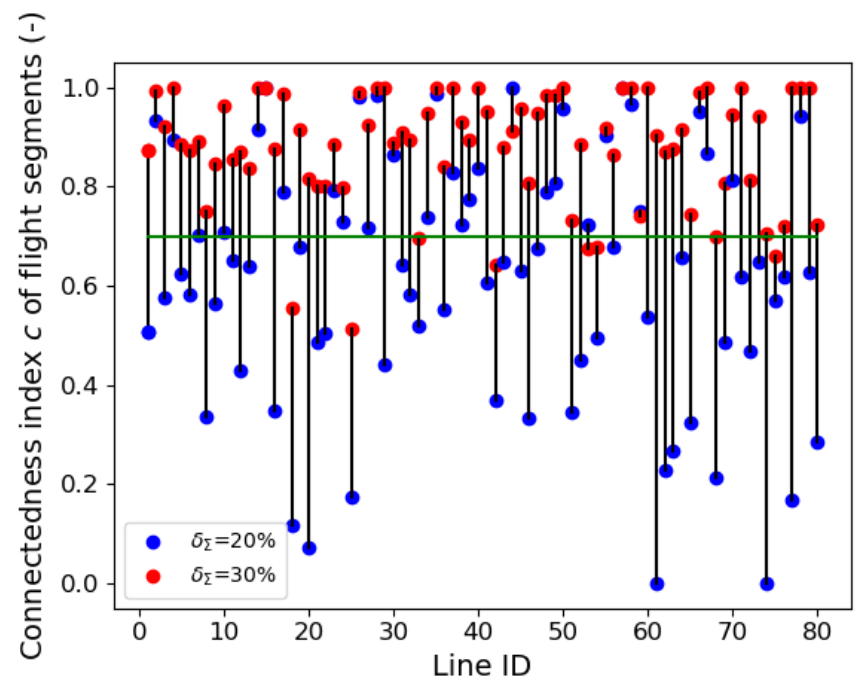

Figure 15. Values of the $c$-index for the 80 lines. Decision threshold $\delta_{c}=0.7$ (green line)

To "validate" the classification obtained by the proposed approach, let us have a look in more details at three lines taken as examples: lines 14, 33 and 59.

\section{- Example of Line 14}

Line 14 has been categorized as Class 1 both for the $L$ and $c$ indexes. This classification highlights a high feasibility for the monitoring mission with long connected flight segments. This can be verified by looking at the bar chart represented in Figure 18. Flight segments identification is shown by considering different combinations of several threshold values: $\quad \delta_{l} \in\{2,5,10\} \mathrm{km}$ and $\delta_{\Sigma} \in\{20,30\} \%$. The cumulative length $L$ of the flight segments is given as a percentage of the line length and as a distance $(\mathrm{km})$. Long connected flight segments have been identified for Line 14, with a low sensitivity to the threshold values. Therefore the relevance of categorization into Class 1 is well verified and long range UAV operations can be performed to monitor this line.

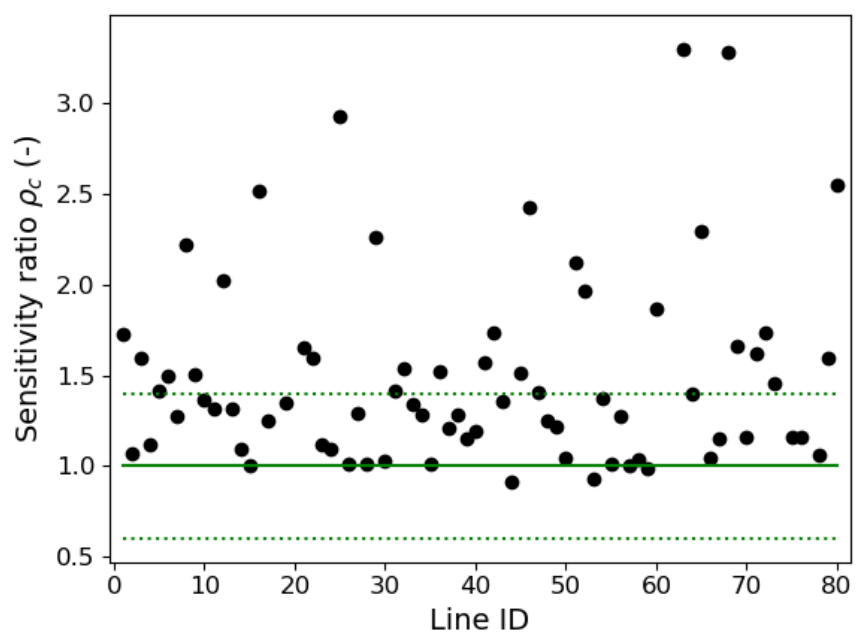

Figure 16. Values of the sensitivity ratio $\rho_{c}$ for the 80 lines. Decision thresholds $\delta_{\rho}{ }^{\min }=0.6$ and $\delta_{\rho}{ }^{\max }=1.4$ (green dotted line)

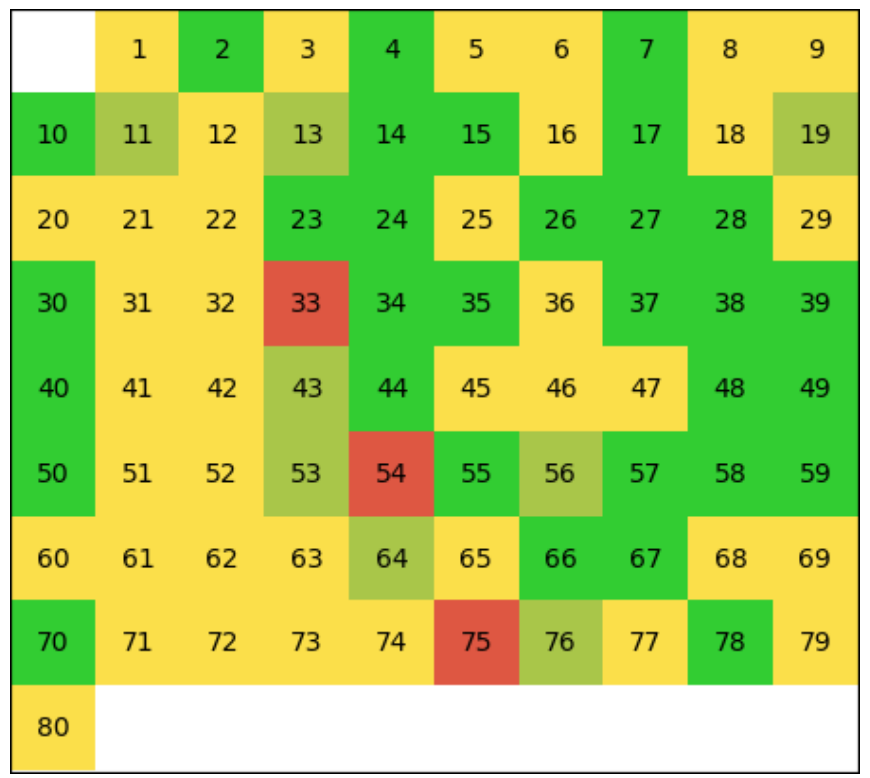

Figure 17. Classification of the lines based on $\left(c, \rho_{c}\right)$. (see Table 1 for color code and definition of the classes)

$\delta_{\Sigma}(\%), \delta_{l}(\mathrm{~km}) \Rightarrow L(\%)=L(\mathrm{~km})$ $20 \mathrm{~s}, 2 \mathrm{~km}=>96.2 \mathrm{~s}=300.0 \mathrm{~km}$

$20 \mathrm{~s}, 5 \mathrm{~km}=>93.6 \mathrm{~s}=292.0 \mathrm{~km}$

$205,10 \mathrm{~km} \Rightarrow 88.1 \%=275.0 \mathrm{~km}$

$205,10 \mathrm{~km}=>88.1 \%=275.0 \mathrm{~km}$

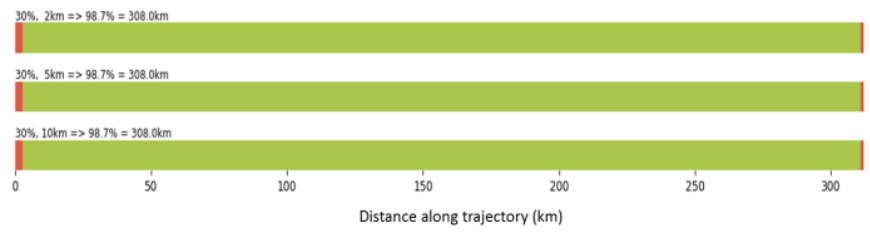

Figure 18. Identified flight segments (in green) along Line 14 


\section{- Example of Line 33}

Regarding the $L$-index, Line 33 has been categorized into Class 2 for $\delta_{L}=50 \mathrm{~km}$, but into Class 3 for $\delta_{L}=100 \mathrm{~km}$, and in Class 4 regarding the $c$-index. This classification can be verified by looking at the bar chart of Figure 19 which shows short sparse flight segments and a significant sensitivity to $\delta_{\Sigma}$. Some short flights may be possible over these segments, but not as an operational long range monitoring mission as studied in this paper.
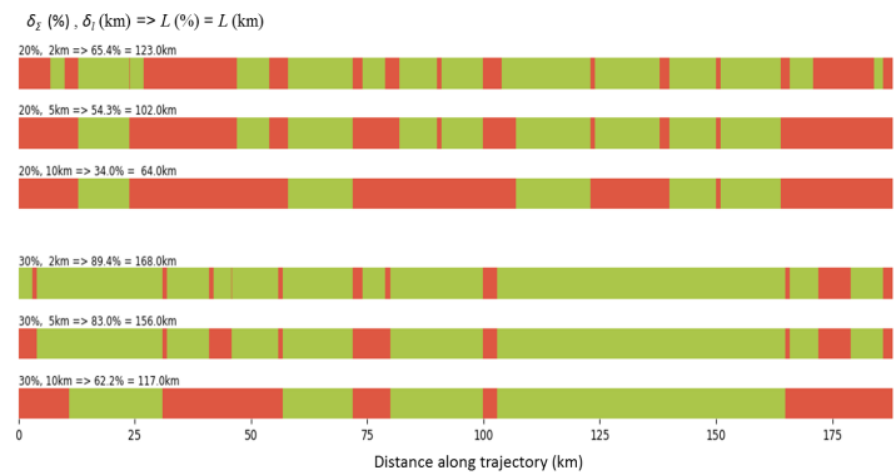

Figure 19. Identified flight segments (in green) along Line 33

\section{- Example of Line 59}

Line 59 has been classified into Class 1 regarding the $L$-index for $\delta_{L}=50 \mathrm{~km}$, but in Class 4 for $\delta_{L}=100 \mathrm{~km}$, and in Class 1 regarding the $c$-index. This indicates well connected flight segments with total length between $50 \mathrm{~km}$ and $100 \mathrm{~km}$. The line being $120 \mathrm{~km}$ long this means that distinct sections are prone to be identified: one section over which flights could be performed and one section over which monitoring operations could not be envisaged. This is confirmed by the bar chart of Figure 20. Note that further investigations would be required to decide whether the feasible section can be covered by only one flight despite the red short segments located around kilometers 75, 85 and 100 which are visible for $\delta_{\Sigma}=20 \%$ (top half part of Figure 20). The risk level map can be analyzed for this purpose and slight modifications of the flight trajectory could be studied in mission preparation. If no modification of the trajectory can be found, several flights will be required to avoid flying over red segments. In this case, deployment could be facilitated by selecting two take-off and landing sites in safe areas located away from the line but close to kilometers 75 and 100. With this strategy, operational flights in both directions would enable to cover the four flight segments of $\sim 10 / 15 \mathrm{~km}$ each.

\section{CONCLUSION}

A method has been proposed in this paper to analyze the feasibility of monitoring mission of infrastructure networks by UAVs. This method enables to identify feasible flight segments over each line of the network, based on risk analysis. Different indexes have been developed as well as a decision making process to produce a comprehensive classification of the lines of the network.

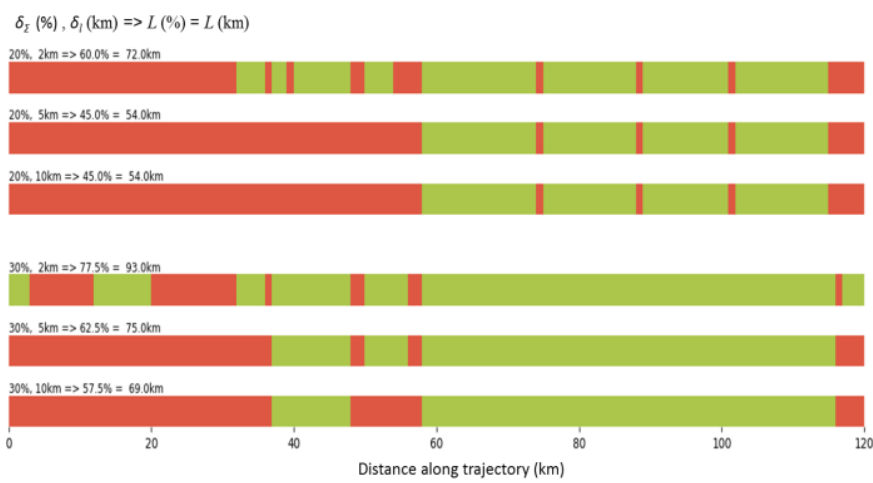

Figure 20. Identified flight segments (in green) along Line 59

This classification takes into account flight regulation and flight operation constraints as well as considerations relative to business interests in asset management. An illustration of the method has been proposed by considering a benchmark of 80 rail lines (representing $17000 \mathrm{~km}$ ) derived from the French railway network.

A direct extension of this work will concern the improvement of the robustness of the proposed approach with respect to its sensitivity to some design or analysis parameters. Additional performance indexes will also be defined to improve the classification process.

In addition to ground risk for people, future work will also consider in the analysis the risk induced for user of road networks (using models proposed by the authors in [6]). Due to their proximity to railways, this is indeed of a particular interest in the feasibility analysis of railway networks but this will also enable to directly apply the proposed method to monitoring missions of road networks by UAVs.

\section{REFERENCES}

[1] M. Bleier, F. Settele, M. Krauss, A. Knoll and K. Schilling, "Risk Assessment of Flight Paths for Automatic Emergency Parachute Deployment in UAVs", IFAC PapersOnLine, vol. 48, no. 9, pp. 180-185, 2015.

[2] L. C. Barr, R. Newman, E. Ancel, C. M. Belcastro, J. V. Foster, J. Evans and D. H. Klyde, "Preliminary Risk Assessment for Small Unmanned Aircraft Systems", 17th AIAA Aviation Technology, Integration, and Operations Conference, 2017.

[3] R. A. Clothier, B. P. Williams and K. J. Hayhurst, "Modelling the Risks Remotely Piloted Aircraft Pose to People on the Ground", Safety Science, vol. 101, pp. 33-47, 2018.

[4] R. Clothier, R. Walker, N. Fulton and D. Campbell, "A Casualty Risk Analysis for Unmanned Aerial System (UAS) Operations over Inhabited Areas," 12th Australian International Aerospace Congress, 2007.

[5] R. Melnyk, D. Schrage, V. Volovoi and H. Jimenez, "A Third-Party Casualty Risk Model for Unmanned Aircraft System Operations", Reliability Engineering and System Safety, vol. 124, pp. 105-116, 2014.

[6] S. Bertrand, N. Raballand and F. Viguier, "Evaluating Ground Risk for Road Networks Induced by UAV Operations", International Conference on Unmanned Aircraft Systems, Dallas, USA, 2018.

[7] S. Bertrand, N. Raballand, F. Viguier and F. Muller, "Ground Risk Assessment for Long-Range Inspection Missions of Railways by UAVs", International Conference on Unmanned Aircraft Systems, Miami, USA, 2017. 
[8] A. La Cour-Harbo, "Quantifying Risk of Ground Impact Fatalities of Power Line Inspection BVLOS Flight with Small Unmanned Aircraft", International Conference on Unmanned Aircraft Systems, Miami, USA, 2017.

[9] K. Dalamagkidis, K. P. Valavanis and L. A. Piegl, "Evaluating the Risk of Unmanned Aircraft Ground Impacts", $16^{\text {th }}$ Mediterranean Conference on Control and Automation, Ajaccio, France, 2008.

[10] K. Dalamagkidis, K. P. Valavanis and L. A. Piegl, "On Integrating Unmanned Aircraft Systems into the National Airspace System", Springer, 2012.

[11] B. Levasseur, S. Bertrand, N. Raballand, F. Viguier and G. Goussu, "Accurate Ground Impact Footprints and Probabilistic Maps for Risk Analysis of UAV Missions", IEEE Aerospace Conference, Big Sky, USA, 2019.

[12] F. J. Gallego, "A Population Density Grid of the European Union", Population and Environment, vol. 31, no. 6, pp. 460-473, 2010.

[13] CORINE Land Cover data set: ttp://www.statistiques.developpementdurable.gouv.fr/donnees-ligne/t/donnees.html

[14] U. C. Kaya, A. Dogan and M. Huber, "A Probabilistic Risk Assessment Framework for the Path Planning of Safe Task-Aware UAS Operations", AIAA SciTech Forum, San Diego, USA, 2019.

[15] E. Rudnick-Cohen, J. W. Herrmann and S. Azarm, "Risk-based Path Planning Optimization Methods for UAVs over Inhabited Areas", ASME 2015 International Design Engineering Technical Conferences \& Computers and Information in Engineering Conference, Boston, USA, 2015. 